\title{
Impact From Dynamic Line Rating on Wind Power Integration
}

\author{
Carl Johan Wallnerström, Member, IEEE, Yalin Huang, Student Member, IEEE, and \\ Lennart Söder, Senior Member, IEEE
}

\begin{abstract}
The concept of dynamic rating (DR) implies that the capacity of a component varies dynamically as a function of external parameters, while the rating traditionally is based on the worst-case. The value of DR thus lies in utilizing existing equipment to a greater extent. By implementing DR and correlating the new ratings with wind power generation, more generation can be implemented. The aim is hence to facilitate connection of renewable electricity production. This paper provides two main contributions: 1) a general dynamic line rating (DLR) calculation model on overhead lines; and 2) an economic optimization simulation model regarding wind power integration comparing DLR with more traditional approaches. These models can both be implemented together, but also separately. The DLR calculation model is easy to use by companies in daily operation where the dynamic line capacity is calculated as a function of static line capacity, wind speed, and ambient temperature. The DLR calculation model is furthermore compared with more comprehensive calculations that validate that the model is accurate enough. This paper also provides an application study where both proposed models are exemplified together and evaluated. Results from this study conclude that it is a significant economic potential of implementing DR within wind power integration.
\end{abstract}

Index Terms-Dynamic line rating, dynamic rating, management decision-making, power distribution, simulation, utilization, wind power.

\section{INTRODUCTION}

M ORE WIND power (WP) generation is expected to be connected at the distribution level and is considered to have the potential of improving system integrity, reliability and efficiency [1]. The challenge is to realize this potential in power distribution. For distribution system operators (DSOs) in countries with a high percentage of WP distributed generation (DG) or a high potential of increasing that percentage, a shared challenge is to integrate WP DG into the current network at low cost and risk. One solution to this challenge is to increase the utilization of the existing system using dynamic rating (DR). Sensing and measurement technology to enable DR is one of the key areas in smart grid development [2].

Traditionally, transfer limits of power components are static, determined using a set of conservative weather conditions and an assumed maximum acceptable conductor

Manuscript received November 29, 2013; revised May 16, 2014; accepted July 7, 2014. Date of publication August 18, 2014; date of current version December 17, 2014. Paper no. TSG-00888-2013.

The authors are with the School of Electrical Engineering, KTH Royal Institute of Technology, Stockholm 10044, Sweden (e-mail: yalin.huang@ee.kth.se).

Color versions of one or more of the figures in this paper are available online at http://ieeexplore.ieee.org.

Digital Object Identifier 10.1109/TSG.2014.2341353 temperature of the component [3]. Usually transfer limits are expressed in terms of ampacity, i.e., the maximum allowable current. Transfer limits of power components obtained in this way are called static rating (SR), while the term static line rating (SLR) is often used when it specifically refers to static rating of power lines. SLR limits ignore both the stochastic and nonstochastic weather impact on the line capacity and correlations to WP generation.

In fact, the line rating depends on the permissible conductor mid-span sag or the maximum sustainable conductor temperature [4]. Both factors are affected by changes in wind, solar, and other parameters [5]. At the same time, the wind power generation has a strong correlation with the weather (temperature, wind condition etc.) as well [6]. To take these factors into account, in this paper DR is proposed for wind power integration, exemplified by analyzing power lines; this sub-category of DR is often referred to as dynamic line rating (DLR).

DLR uses direct, real-time measurements of conductor temperature or real-time estimates of the temperature using actual weather conditions [7]. The application of DLR will provide the true transfer capacity of the grid in real time, gain generation capacity in the network and improve the utilization of the existing system [5], [8]-[10]. This paper proposes a new general DLR calculation model based on standard capacity calculations, but simplified and accurate to be implemented in the daily operation. The DLR calculation model provides the dynamic line capacity as a function of static line capacity, wind speed, and ambient temperature.

Although the DLR calculation model focuses on transfer limits of overhead conductors, DR is highly relevant to implementation on other power components that can act as transfer bottlenecks. For example, much research and development has focused on using DR for power transformers [11]. Moreover, the economic aspect of applying DR in a power system must be justified [12]. In order to integrate much wind power as long as that is economically motivated, the present paper also develops an economic optimization simulation model regarding wind power integration comparing DLR with more traditional approaches. Most importantly, the proposed simulation model considers the correlation between line capacity and wind power production. This is represented through the dependency of each of these factors on weather and wind speeds. However, the proposed simulation model can be adjusted to include other DR power components as input. In the proposed simulation model, life cycle costs analysis (LCCA) including costs and economic benefits (with negative signs) is applied. 
In addition, it outputs the curtailed wind power due to the line capacity limits in each case to evaluate the risk. In this paper, this curtailed energy is referred to as disconnected energy not served (DiscENS). It can be considered as costs for the wind power plants depending on the regulation.

The proposed DLR model is compared with more comprehensive calculations that validate the model is accurate enough. And the simulation results show that applying DLR is more significantly advantageous than SLR when there are more wind power plants. Moreover, the disconnected energy due to line limits increases with the number of wind power plants. Applying DLR, more energy will be used than applying SLR.

\section{Dynamic Line Rating Calculation}

This section develops a general DLR calculation model based on the standard line capacity calculation, but simplified and easy to be implemented. It is verified with examples later in Section VI.

\section{A. Standard Line Capacity Calculation}

IEEE standard 738 [13] gives a standard formula for calculating the current of bare overhead conductors. It is based on steady-state heat balance

$$
I_{\max }=\sqrt{\frac{q_{c}+q_{r}-q_{s}}{R\left(T_{c}\right)}}
$$

where $q_{c}$ is the convective cooling, $q_{r}$ is the radiative cooling, and $q_{s}$ is the solar heating. $R\left(T_{c}\right)$ is the resistance of the conductor at temperature $T_{c}$. Solar radiation is neglected as recommended in [14]. The largest resistance of the line (under the highest allowed conduction temperature) is used to reduce the risk of the line exceeding its thermal rating. The line capacity can be calculated as below [13]

$$
\left\{\begin{array}{l}
\frac{\sqrt{\left[1.01+0.0371 *\left(\frac{D * \rho_{f} * v}{\mu_{f}}\right)^{0.52}\right] *\left[k_{f} * K_{\text {angle }} * \Delta T\right]}}{\sqrt{R\left(T_{c}\right)}} \\
\frac{\sqrt{\left[0.0119\left(\frac{D * \rho_{f} * v}{\mu_{f}}\right)^{0.6}\right] *\left[k_{f} * K_{\text {angle }} * \Delta T\right]}}{\sqrt{R\left(T_{c}\right)}}
\end{array}\right.
$$

where $\Delta T=T_{c}-T_{a} . D$ is the conductor diameter, $\rho_{f}$ is the density of air at temperature $T_{f}\left(T_{f}=\left(T_{c}+T_{a}\right) / 2\right.$, where $T_{c}$ and $T_{a}$ are conductor temperature and ambient air temperature, respectively), $v$ is the speed of air stream at conductor, $\mu_{f}$ is the dynamic viscosity of air at temperature $T_{f}, k_{f}$ is the thermal conductivity of air at temperature $T_{f}, K_{\text {angle }}$ is a parameter represents the angle between wind speed and the conductor axis. The formula above corresponds to low wind speeds, while the one below corresponds to high wind speeds. However, at any wind speed, the larger of the two values is used [13].

The line capacity in reality is varying with many parameters as shown in (2), however, it is useful to simplify it to be easily implemented for electric power system planning and operation. In general, there are two ways to simplify the capacity calculation, SLR, and DLR.

\section{B. Calculation of SLR}

SLR needs the parameters from a scenario, which usually is the worst scenario considering the worst possible conductor temperature, the ambient air temperature and the wind speed. For example, engineering recommendation (ER) P27 [14] proposes to calculate seasonal thermal ratings using assumed temperatures for different seasons and for a constant wind speed of $0.5 \mathrm{~m} / \mathrm{s}$ and zero solar radiation [10]. The conventional relations between $T_{f}, \mu_{f}, \rho_{f}$, and $k_{f}$ can be found in Standard 738 [13]

$$
\frac{\sqrt{\left[1.01+0.0371 *\left(\frac{D * \rho_{f} * v_{\max }^{\mathrm{SLR}}}{\mu_{f}}\right)^{0.52}\right] *\left[k_{f} * K_{\text {angle }}^{\mathrm{SLR}} * \Delta T^{\mathrm{SLR}}\right]}}{\sqrt{R^{\mathrm{SLR}}}}
$$

where $v^{\mathrm{SLR}}$ is the wind speed, $\Delta T^{\mathrm{SLR}}$ is the temperature difference, $R^{\mathrm{SLR}}$ is the line resistance used in the scenario of SLR calculation. Other parameters depends on $T_{f}$ also correspond to the scenario of SLR calculation.

\section{Proposed Calculation of DLR}

DLR estimates the line ampacity in real time with monitored weather conditions taking account of the wind cooling effect [15]. It can be obtained using a variety of methods: conductor sag and tension monitoring, vibration mode analysis, conductor temperature and local weather data [4], [15]. Many trials have been carried out on using online measurement and communicate with the DSO or the devices to set the realtime line capacity [16]-[19]. Temperature and wind speed are the parameters that affect DLR significantly, furthermore, the higher the wind speed, the higher the wind power production and simultaneously transfer capacity. As for the correlation between temperature and the wind speed are unclear, their impact on capacity ratio are studied separately. This paper develops a simplified DLR calculation model on the assumption that wind impact on the line rating and temperature impact on that are independent. This is demonstrated as a reasonable assumption with numerical example in Tables IV and $\mathrm{V}$ in Section VI.

The proposed DLR model is based on the capacity ratio between DLR and SLR. This ratio $(\eta)$ is a function of wind speed and temperature, as shown in (4). The variation of resistance due to temperature is neglected for the line capacity calculation in this paper. Under the independence assumption, $\eta_{v}$ and $\eta_{T}$ are obtained separately

$$
\eta(v, T)=\frac{I_{\max }^{D L R}}{I_{\max }^{\mathrm{SLR}}} \approx \eta_{v} * \eta_{T}
$$

where $\eta_{v}$ is the ratio related to the wind speed, and $\eta_{T}$ is the ratio related to the relevant temperature values. Given the same temperature for DLR and SLR calculation, all the temperature related parameters $\left(\rho_{f}, \mu_{f}, k_{f}\right.$, and $\left.\Delta T\right)$ are the same for both cases. We also use the wind speed $v$ that is perpendicular to the conductor, so the wind direction factor $K_{\text {angle }}$ is equal to 1 . The parameters to define $\eta_{v}$ can be estimated for both low wind speed $\left(\eta_{\text {low }}(v)\right)$ and high wind speed $\left(\eta_{\text {high }}(v)\right)$. Note that even 
though the wind speed is very low, the line rating ratio should not be lower than 1

$$
\left\{\begin{array}{l}
\eta_{v}=\max \\
\eta_{\text {low }}(v) \approx \frac{1}{v_{\mathrm{SLR}}^{0.26}} * v^{0.26}=\alpha * v^{0.26} \\
\eta_{\text {high }}(v) \approx \frac{0.566}{v_{\mathrm{SLR}}^{0.26}}\left(\frac{\rho_{f}}{\mu_{f}}\right)^{0.04} D^{0.04} v^{0.3}=\beta * D^{0.04} * v^{0.3}
\end{array}\right.
$$

where $\alpha=v_{\mathrm{SLR}}^{-0.26}$ and $\beta=\left(0.559 / v_{\mathrm{SLR}}^{0.26}\right)\left(\rho_{f} / \mu_{f}\right)^{0.04}$. Conventional $v_{\mathrm{SLR}}=0.5$, then $\alpha \approx 1.1975$.

If we further assume that some of the temperature related parameters, $\rho_{f}, \mu_{f}$, and $k_{f}$, are the same in the DLR and SLR calculation. Therefore, the value of $\beta$ is set by the $T_{f}$ in SLR calculation. $\eta(v, T)$ is derived as

$$
\left\{\begin{array}{l}
1 \\
\approx \eta_{\text {low }}(v) \sqrt{\frac{T_{c}-T_{A}}{T_{c}-T_{A}^{\mathrm{SLR}}}}=\eta_{\text {low }}(v) \eta_{T} \\
\approx \eta_{\text {high }}(v) \sqrt{\frac{T_{c}-T_{A}}{T_{c}-T_{A}^{\mathrm{SLR}}}}=\eta_{\text {low }}(v) \eta_{T}
\end{array}\right.
$$

where $\eta_{T}$ can be expressed as

$$
\eta_{T} \approx \sqrt{\frac{T_{c}-T_{A}}{T_{c}-T_{A}^{\mathrm{SLR}}}}=\gamma \Delta T^{0.5}
$$

where $\gamma=\sqrt{1 /\left(T_{c}-T_{A}^{\mathrm{SLR}}\right)}$ and $\Delta T=T_{c}-T_{A}$.

\section{Simulation Model}

This section proposes an economic optimization simulation model regarding wind power integration. This proposed simulation model applies the developed DLR model to analyze impact from DLR on WP connection. However, it is also possible to apply other DR models. The main inputs for the analysis are the dynamic parameters (e.g., wind speed and the ambient temperature). Two outputs produced from the analysis are LCCA and curtailed wind power which can be used for the power distribution decision-making. The analysis shows how the optimal number of integrated WP plants and the disconnected energy due to the transfer limitations are affected.

\section{A. $L C C A$}

Life cycle cost (LCC) is the annualized cost of an investment during its entire economic lifetime. LCC analyzes (LCCA) often includes costs of investments, outages, maintenance, interests and incomes (modeled as negative costs) [20]. The estimated lifetime is limited by the technical lifetime (LT), technical developments and expansions in power distribution systems [20]. The LCC of a wind power plant investment LCC $^{\text {inv }}$ without considering the incomes is performed as follows before each simulation:

$$
\mathbf{L C C}^{i n v}=\text { Capex }+\sum_{L T} \frac{\text { Opex }_{i}}{q^{i}}-\frac{R}{q^{L T}}
$$

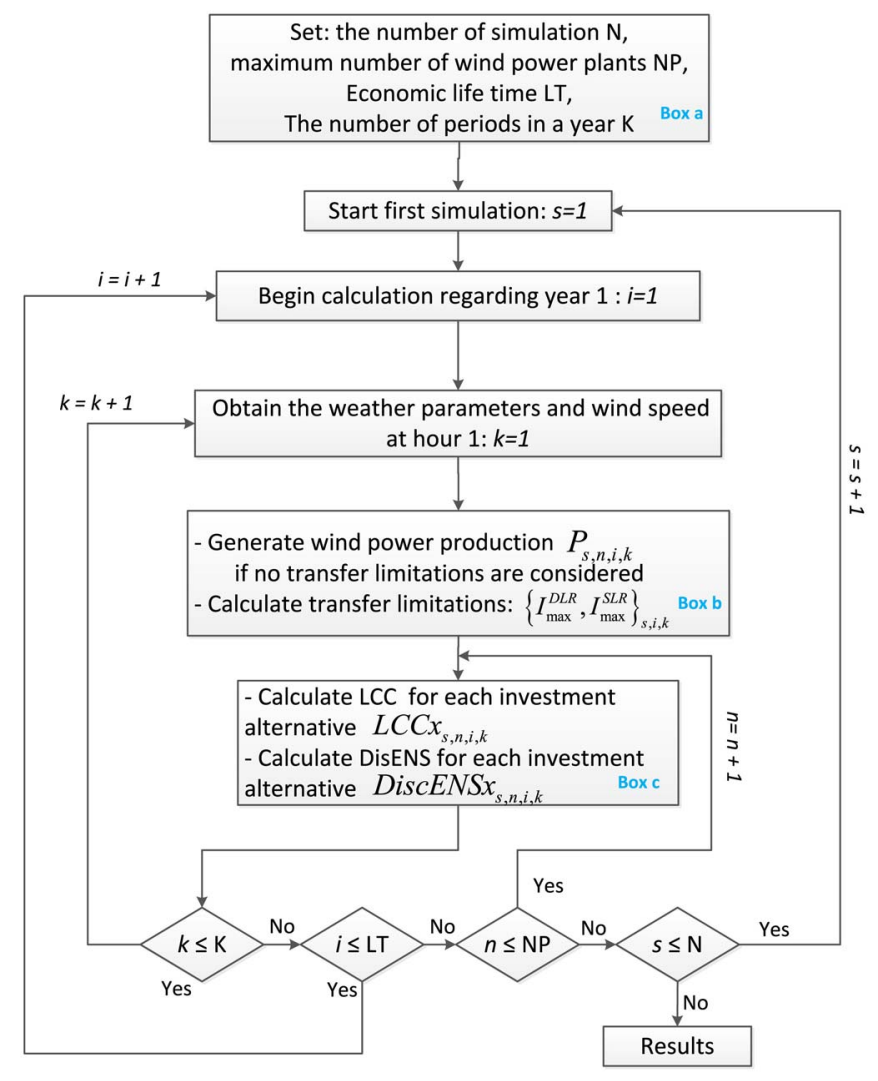

Fig. 1. Model for assessing wind power connection using SLR and DLR.

$$
q=1+z
$$

where Capex represents the capital investment, Opex Opepre- $^{-}$ sents the operational expense in year $i$, all the operational expenses are discounted to the first year which is the capital investment year by using the discount factor $q_{i}$. This is called the net present value (NPV) method. This factor is depend on the discount interest rate $z . R$ represents the residual value of the investment after its technical LT.

\section{B. Method}

The flow chart of the method is shown in Fig. 1. First, as shown in Box a, the number of simulations $(\mathrm{N})$, the maximum number of wind power plants (NP), the economic life time of the power plants (LT) and the number of periods in a year $(\mathrm{K})$ are set. For example, $K$ is set to 8760 for hourly analysis in a year. Hence, there are $N * L T * K$ generations of stochastic variables for a certain number of WP plants.

Then, as shown in Box b, dynamic (temperature and wind power production) inputs are generated in each simulation. To save time without losing any generality, the same stochastic variables are used for generation calculation for each WP plant and DLR calculation regarding different numbers of WP plants. In this model, wind power production is assumed to be correlated only to wind speed and not to temperature. By comparing the generated wind power and the line limits, the permissive transferred power is obtained.

The income from transferred power is considered as negative costs. It is discounted to NPV as operational costs. 
Therefore, the LCC for the investment, Box c, is calculated as follows:

$$
\begin{aligned}
\operatorname{Inc}_{s, n, i, k}= & \min \left(P_{s, n, i, k}, \widehat{P}_{s, i, k}\right) * E_{\text {price }} \\
\widehat{P}_{s, i, k}= & \widehat{I}_{s, i, k} * U_{N} \\
& \widehat{I}_{s, i, k} \in\left\{I_{\max }^{D L R}, I_{\max }^{\mathrm{SLR}}\right\}_{s, i, k} \\
\mathrm{LCC}_{s, n, i, k}= & \operatorname{LCC} x^{i n v}-\frac{1}{(1+z)^{i}} * \operatorname{Inc} c_{s, n, i, k} \\
\operatorname{LCC} x_{s, n}= & \operatorname{LCC} x^{i n v}-\sum_{i=1}^{L T} \frac{1}{(1+z)^{i}} \sum_{k=1}^{12} \operatorname{Inc}_{s, n, i, k}
\end{aligned}
$$

where $k$ represents any period in a year, $i$ represents any year during LT, $n$ denotes the number of the wind farms, $s$ presents any simulation. $\widehat{P}_{s, i, k}$ is the transmission limit on the connection line, which is calculated by the maximum line capacity (either calculated by DLR model $I_{\max }^{\mathrm{DLR}}$ or SLR model $I_{\max }^{\mathrm{SLR}}$ ) and nominal voltage $\left(U_{N}\right)$ at the connection point of wind power plants. This value is not dependent on the number of wind power plants but it is related to the line rating model. $P_{s, n, i, k}$ presents the possible wind power generation in the simulation $s$ with $n$ wind power plants in the period $k$ in year $i$. The smaller value between these two values represents the transmitted wind power, either all the wind power is transmitted or curtailed due to the transmission limits. The transmitted power multiply the electricity price gives the income for the wind power plants $\left(I n c_{s, n, i, k}\right)$. This is discounted by NPV method to evaluate different investment alternatives. $x$ represents different investment alternatives. For example, investment on a line or on wind power plants. If the average LCC is negative at the end of the economic lifetime, this indicates that the investment is profitable.

\section{Disconnected Energy Not Served (DiscENS)}

The amount of DiscENS is recorded in each simulation, as shown in Box c, if it is applicable. It reflects the utilization of the network and the renewable energy. It is assumed that the wind power plants can be disconnected due to the limited line rating. So when wind power plants produce more than the limit, the extra energy will be curtailed. In the model, the production of the wind power plants is first generated without considering the transmission limits, then the sum of curtailed power is calculated according to the limits

$$
\operatorname{DiscENS} S_{s, n, i, k}=\max \left(P_{s, n, i, k}, \widehat{P}_{s, n, i, k}\right)-P_{s, n, i, k} .
$$

If the transmission limit is larger than the produced wind power, the curtailed power is the difference between them; otherwise the curtailed power is zero. Although costs of introducing DLR are not considered in this simulation model. However, by calculating the difference in profit between SLR and DLR, it reveals the highest cost allowed for introducing DLR.

\section{ApPliCATION}

This section describes an implemented application study to evaluate both the DLR calculation model and the simulation model that are proposed in this paper. The whole simulation is repeated several times until the results converge.

\section{A. Background}

The performance of the proposed simulation model is demonstrated by a small example in a windy area with one connection point to a distribution network. The bottleneck of integrating more wind power plants is the connection line between this windy area and the connection point in the distribution network. This application study aims to identify the optimal number of WP plants in the network when SLR or DLR is used, and the level of utilization of the network as well as the curtailed wind power. Different line rating models are applied in this case study to show the advantages of the proposed DLR model.

As the amount of wind power increases, the existing network faces the challenge to host it. In this case study, the profits of installing another 1 to 7 wind power plants are investigated and stochastic variables are generated hourly. The DSO is obliged to connect customers (consumers and producers). However, the producer will pay for the investments needed for the connection. Hence, it is necessary for WP owners to investigate cost analysis on the possible ways to connect WP plants: whether to build a new line, or to apply DLR on the connection lines. On the one hand, extending the capacity of the lines (building one extra line) can be challenging in a reasonable time frame and budget and to refuse the connection of new wind farms is not an option. In some areas, it is increasingly difficult to build additional lines or even physical upgrading the old lines. On the other hand, DLR calculation is rather complex. And the difficulty is to make all power system players agree on and allow the risks.

\section{B. Input Data}

Values of monthly average temperature and wind data that are typical for mid-west part of Sweden (Värmland mid-west Sweden) between year 1970 and 2013 are used, as shown in [21]. The standard deviation of the monthly average temperature is assumed to be $5^{\circ} \mathrm{C}$. The total cost includes the cost of constructing and operating wind power plants, the cost of an extra line (if it is applicable), and the income (modeled as a negative cost) from selling electricity. The operation and maintenance costs of a WP plant and a line are discounted to the first year of the LT and are considered in $\mathrm{LCC}_{1 w p p}^{i n v}$ and $\mathrm{LCC}_{1 \text { line }}^{\text {inv }}$, respectively. The input parameters are listed below:

$N P=7$;

$K=8760$;

$L T=20$ years;

$z=7 \%$

$P_{\max }=3 \mathrm{MW}$;

$D=28 \mathrm{~mm}$

$T_{C}^{\mathrm{SLR}}=70^{\circ} \mathrm{C}$;

$T_{a}^{\mathrm{SLR}}=35^{\circ} \mathrm{C}$;

$E_{\text {price }}=569 \mathrm{SEK} / \mathrm{MWh}$;

$\mathrm{LCC}_{1 w p p}^{i n v}=15000000$ SEK;

$\mathrm{LCC}_{1 \text { line }}^{\text {inv }}=20000000$ SEK.

$P_{\max }$ is the power capacity of each WP plant. The correlation between electric energy consumption and wind speed has been calculated in [20] using Swedish data, and it is only 0.02. Therefore, the electricity price is assumed fixed. It includes 
TABLE I

ANNUAL Distribution of Wind SPEEDS AND WIND POWER PRODUCTION

\begin{tabular}{lllll}
\hline \hline $\begin{array}{l}\text { Wind } \\
\text { Speed } \\
\begin{array}{l}\text { Intervals } \\
(\mathrm{m} / \mathrm{sec})\end{array}\end{array}$ & $\begin{array}{l}0.2^{*} \text { Wind } \\
\text { Speed } \\
\text { Intervals } \\
(\mathrm{m} / \mathrm{sec})\end{array}$ & $\begin{array}{l}\text { produced } \\
\text { power/power } \\
\text { capacity } \\
(\%)\end{array}$ & $\begin{array}{l}\eta \\
\text { Equ.2and3 } \\
(\%)\end{array}$ & $\begin{array}{l}\eta \\
\text { Equ.5(\%) }\end{array}$ \\
\hline $0-0.75$ & 0.50 & 0.00 & 0 & 0 \\
$0.75-2.25$ & 0.50 & 0.05 & 0 & 0 \\
$2.25-3.75$ & 0.50 & 1.23 & 0 & 0 \\
$3.75-5.25$ & 0.75 & 6.67 & 110.9 & 111.1 \\
$5.25-6.75$ & 1.05 & 15.95 & 120.8 & 121.3 \\
$6.75-8.25$ & 1.35 & 33.16 & 129.5 & 129.5 \\
$8.25-9.75$ & 1.65 & 57.00 & 137.5 & 137.6 \\
$9.75-11.25$ & 1.95 & 87.17 & 144.6 & 144.6 \\
$>11.25$ & 2.35 & 100 & 152.9 & 153.0 \\
\hline
\end{tabular}

two sources: the value for directly selling the energy and the tariff of green certificate for producing renewable energy. The combined price is based on historical data, as used in [22]. Nordpool [23] is the reference for the selling price of energy and the Swedish national grid [24] for the green certificate price.

The stochastic input variables are generated as follows.

1) Temperature: Temperature data are sampled every hour. They are assumed to be normally distributed and based on hourly history data in the same Swedish county during 48 years, where the wind speed data is also obtained. The temperature average is modeled as a function of both month and hour. The value directly influences the line limits if DLR is applied according to (7). Moreover, the incomes from the wind farms are related to permitted wind power production.

2) Wind Power Production: Wind power production is modeled as a function of wind speeds. Different wind speed bands with associated average production are shown in Table I, which are based on the values in [22]. The production of a WP plant is produced according to Table I and $P_{\max }$. It is assumed that the wind speed in each period in a year is independent and only $20 \%$ of it is perpendicular to the lines. A uniform distribution random number is generated in each simulation to model the probability. Subsequently the corresponding wind power generation is obtained.

\section{Calculation of SLR}

In this paper, the worst case is assumed when the wind speed is $0.5 \mathrm{~m} / \mathrm{s}$ (the equation for low wind speeds is used) and the temperature is $35^{\circ} \mathrm{C}$ (this of course can be different in different countries). Since $v$ is the wind speed perpendicular to the conductor axis, $K_{\text {angle }}$ is equal to one. SLR applied on the existing network to identify the wind power transmission limit is the method referred to as SLR 1, while building an extra connection line in order to increase the transmission capacity (which is still based on SLR) is referred to as SLR 2.

\section{Calculation of DLR}

As it is problematic to know how the wind speed in a line corridor correlates with the wind speed hitting the wind power plant. Hence the contribution from wind speed has to be much

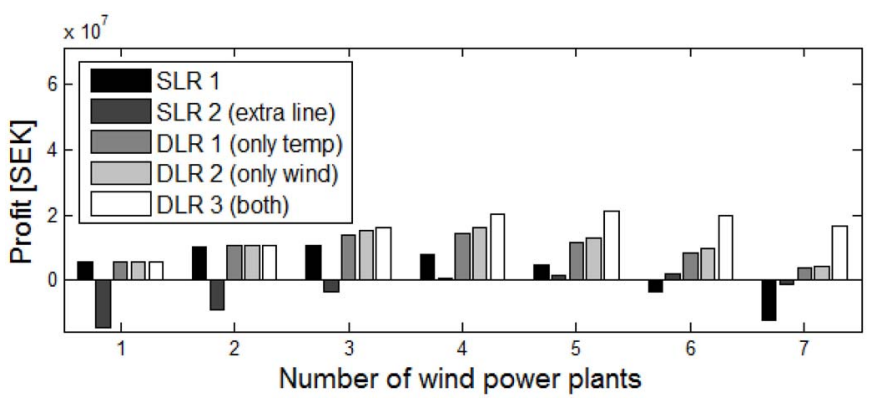

Fig. 2. Total profits using different investment alternatives.

more pessimistic compared with the theoretical value based on the wind speed hitting the wind power plant. Therefore, $20 \%$ (but never lower than $0.5 \mathrm{~m} / \mathrm{s}$ ) of the wind speed hit the conductor hot spot is assumed. Furthermore the calculations are based on the lower part of each wind speed interval in the first column of Table I.

In this case study it is found that if the wind speed is less than $1.34 \mathrm{~m} / \mathrm{s}$ it is considered as low wind speed, i.e., when the wind speed is lower than $1.34 \mathrm{~m} / \mathrm{s}$, it is always applicable to use the low wind speed formula in (2), and when the wind speed is higher than $1.34 \mathrm{~m} / \mathrm{s}$, it is always applicable to use the high wind speed formula in (2).

In addition, two variants of the proposed DLR calculation model presented in Section II are also implemented.

1) A simplified version referred to as $D L R \quad 1$ when only the temperature impact on line rating is considered as in (7).

2) A simplified version referred to as $D L R 2$ when only the wind impact on line rating is considered as in (5).

3) The proposed DLR model is referred to as $D L R 3$ which takes the temperature and wind speed impact on line rating into consideration as in (6).

These three models are compared to show the value of a complex DLR model considering both wind speed and temperature impact.

\section{Results}

Five alternatives for the DSO are investigated, Four aspects of these alternatives are analyzed. First the different profits by different alternatives with an increase of the number of wind power plants are presented. Then the DiscENS values are compared with the increase of wind power plants. After that the optimal wind power plants to be connected to the grid is identified. Last the value or the price of introducing DLR is presented.

\section{A. Number of Simulations}

The number is set to 500 for this case study. To validate that this give accurate results, the results from 100 and 250 simulations are compared. After 250 simulations, the results (average, max, and min values) are almost the same as after 500 simulations, but already at after 100 simulations the results are close to that. If only averages values and the optimal amount of wind power is of interest, lower number of simulations are needed compared if finding extreme values are of interest. 


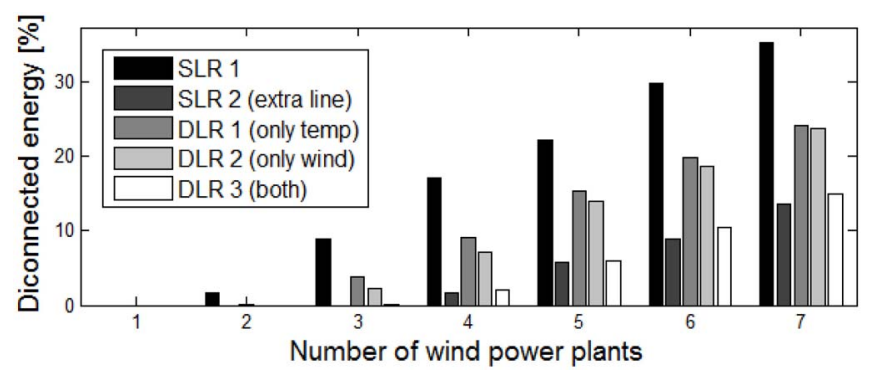

Fig. 3. Disconnected wind power [\%] using different investment alternatives.

\section{B. Total Profits}

As the number of WP plants increases, the profits first increase and then decrease in any investment alternative as shown in Fig. 2. Applying SLR 1 in the existing network is profitable until the number of WP plants reaches six. Applying SLR 2 becomes profitable when the number of wind power plants exceeds four. This investment alternative is not that attractive since even the maximum possible profit is very low. Comparing the profits of applying $D L R ~ 1, D L R 2$, and $D L R 3$, the profit of applying $D L R 3$ is always the highest. The difference increases as the number of installed wind power plants. This shows that the if only one element is considered in DLR estimation, the potential of DLR is much underestimated.

\section{DiscENS Comparison}

The disconnected energy due to transfer limitations increases with the number of WP plants, as shown in Fig. 3. It is always the option applying SLR 1 that leads to the highest DiscENS. While the lowest DiscENS happens in applying SLR 2 or DLR 3. DLR 3 has the lowest discENS among all the DLR models. This shows that the proposed DLR model increases the utilization of the network. Moreover, the DiscENS in SLR 1 option is much higher than that using SLR 2. This shows that building an extra line can decrease the DiscENS more efficiently than DLR option and increase the utilization of renewable energy significantly in this case.

\section{Optimal Amount of Wind Power}

The optimal number of wind power plants for four investment alternatives can be observed from Fig. 2 as well. Table II summarizes the optimal decision. Using $D L R 3$ can result the highest profit without considering the cost of introducing DLR. This shows that the optimal investment alternative is dependent on the potential of wind power resource or the wind power plants connection.

\section{E. Value of $D L R$}

The cost of applying DLR is not included in the profit calculation. Therefore, the difference between their profits, which reveals the value of implementing DLR (or the maximum allowed cost of implementing DLR), is more relevant. It can be seen that the value of DLR is increasing as the number of WP plants increases. Fig. 4 shows different profits between DLR and SLR 1. The difference reflects the value of introducing DLR. In any model of DLR this value increases with the

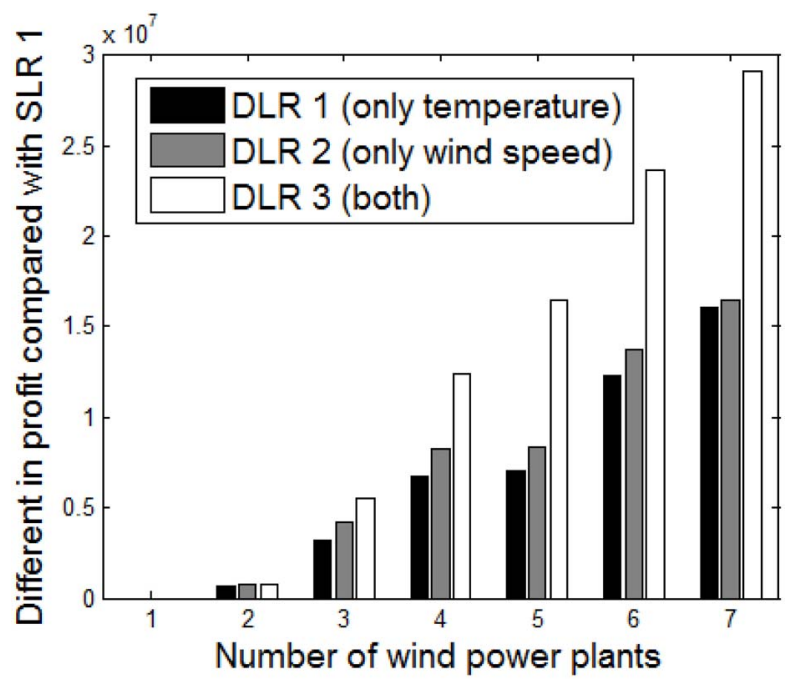

Fig. 4. Value of applying DLR.

number of WP plants. When the WP plants are fewer, the value of using DLR is less. Introducing DLR 3 has higher value than $D L R 2$ and $D L R 1$. This value can be used to evaluate whether it is profite to install devices for DLR.

\section{F. General Analysis}

With DLR, the limits of the lines are more accurate and better utilization of the network can lead to benefits. Consequently, the existing system can host more wind power without extension. The wind power plant owners can also export more power, consumers can purchase more green energy and the network owner can defer reinforcement of, and new investment in, lines. In other words, the same amount of wind power can be hosted with fewer and/or thinner power lines. On the other hand, sensors and other equipment have to be installed and a lot of data have to be managed in control rooms. Another possible disadvantage with DLR compared with installation of a new line of higher capacity is higher losses, because the same amount of power then is transported on a line with higher resistance. The control room and sensor issues can, however, be easier to solve using a simplified, but still accurate, DLR model as proposed in this paper.

\section{VAlidation of the PRoposed DLR Calculation Model}

$D=28 \mathrm{~mm}$ and $T_{c}=70^{\circ} \mathrm{C}$ are set in the validation process. First the assumption of independence of wind speed and temperature impact on DLR is validated. Then the accuracy of the ratio calculation compared with the comprehensive DLR model is presented. Finally, the implementation of proposed DLR models are compared with the comprehensive DLR model in the same case study.

Given the same conservative $T_{c}$ for both in SLR and DLR calculation, the DLR ratio $\eta$ can be calculated for different wind speeds and ambient temperature, as shown in Table III. The ratio is calculated by the comprehensive formula illustrated in (2) and (3). 
TABLE II

MaXimum Profits and Optimal Number of WP Plants Using DifFerent InVESTMEnt Alternatives

\begin{tabular}{ccccccc}
\hline \hline Option & Optimal nr. & Aver. & $\begin{array}{c}\text { Profit(SEK) } \\
\max \end{array}$ & $\min$ & Second best nr. & $\begin{array}{c}\text { Profits(SEK) } \\
\text { Aver. }\end{array}$ \\
\hline SLR 1 & 3 & $1.09 * 10^{7}$ & $1.16 * 10^{7}$ & $1.03 * 10^{7}$ & 2 & $1.02 * 10^{7}$ \\
SLR 2 & 6 & $0.19 * 10^{7}$ & $0.31 * 10^{7}$ & $0.05 * 10^{7}$ & 5 & $0.15 * 10^{7}$ \\
DLR 1 & 4 & $1.45 * 10^{7}$ & $1.53 * 10^{7}$ & $1.36 * 10^{7}$ & 3 & $1.41 * 10^{7}$ \\
DLR 2 & 4 & $1.61 * 10^{7}$ & $1.70 * 10^{7}$ & $1.52 * 10^{7}$ & 3 & $1.51 * 10^{7}$ \\
DLR 3 & 5 & $2.12 * 10^{7}$ & $2.22 * 10^{7}$ & $2.00 * 10^{7}$ & 4 & $2.03 * 10^{7}$ \\
\hline
\end{tabular}

TABLE III

TABle of $\eta(\%)$ At DifFERENT Wind SPEed AND TEMPERATURE

\begin{tabular}{l|l|l|l|l|l|l|l}
\hline$\eta(\%)$ & \multicolumn{7}{|c}{$T_{a}\left({ }^{\circ} \mathrm{C}\right)$} \\
\hline$v(m / s)$ & 35 & 25 & 15 & 5 & -5 & -15 & -25 \\
\hline 0.5 & 100.0 & 113.1 & 124.9 & 135.7 & 145.7 & 155.1 & 164.0 \\
1.0 & 119.3 & 135.1 & 149.3 & 162.2 & 174.2 & 185.5 & 196.2 \\
2.0 & 145.7 & 165.5 & 183.2 & 199.5 & 214.7 & 228.9 & 242.4 \\
3.0 & 164.6 & 165.2 & 165.6 & 166.0 & 166.3 & 166.6 & 166.9 \\
4.0 & 179.4 & 203.7 & 225.6 & 245.6 & 264.3 & 281.8 & 298.5 \\
5.0 & 191.8 & 217.8 & 241.2 & 262.6 & 282.6 & 301.4 & 319.2 \\
6.0 & 202.6 & 230.1 & 254.8 & 277.4 & 298.5 & 318.3 & 337.1 \\
\hline
\end{tabular}

TABLE IV

$\eta(\%)$ AS A FUNCTION OF $v$

\begin{tabular}{c|c|c|c|c|c|c|c}
\hline$\eta(\%)$ & \multicolumn{7}{|c}{$T_{a}\left({ }^{\circ} \mathrm{C}\right)$} \\
\hline$v(m / s)$ & 35 & 25 & 15 & 5 & -5 & -15 & -25 \\
\hline 0.5 & 100.0 & 100.0 & 100.0 & 100.0 & 100.0 & 100.0 & 100.0 \\
1.0 & 119.3 & 119.4 & 119.5 & 119.5 & 119.5 & 119.6 & 119.6 \\
2.0 & 145.7 & 146.3 & 146.7 & 147.0 & 147.3 & 147.6 & 147.8 \\
3.0 & 164.6 & 165.2 & 165.6 & 166.0 & 166.3 & 166.6 & 166.9 \\
4.0 & 179.4 & 180.1 & 180.6 & 181.0 & 181.3 & 181.7 & 182.0 \\
5.0 & 191.8 & 192.5 & 193.1 & 193.5 & 193.9 & 194.2 & 194.6 \\
6.0 & 202.6 & 203.4 & 203.9 & 204.4 & 204.8 & 205.2 & 205.5 \\
\hline
\end{tabular}

TABLE V

$\eta(\%)$ AS A FUNCTION OF $T_{a}$

\begin{tabular}{l|l|l|l|l|l|l|l}
\hline$\eta(\%)$ & \multicolumn{7}{|c}{$T_{a}\left({ }^{\circ} \mathrm{C}\right)$} \\
\hline$v(m / s)$ & 35 & 25 & 15 & 5 & -5 & -15 & -25 \\
\hline 0.5 & 100.0 & 113.1 & 124.9 & 135.7 & 145.7 & 155.1 & 164.0 \\
1.0 & 100.0 & 113.2 & 125.1 & 136.9 & 146.0 & 155.5 & 164.4 \\
2.0 & 100.0 & 113.6 & 125.7 & 136.9 & 147.3 & 157.1 & 166.4 \\
3.0 & 100.0 & 113.6 & 125.7 & 136.9 & 147.3 & 157.1 & 166.4 \\
4.0 & 100.0 & 113.6 & 125.7 & 136.9 & 147.3 & 157.1 & 166.4 \\
5.0 & 100.0 & 113.6 & 125.7 & 136.9 & 147.3 & 157.1 & 166.4 \\
6.0 & 100.0 & 113.6 & 125.7 & 136.9 & 147.3 & 157.1 & 166.4 \\
\hline
\end{tabular}

\section{A. Wind Speed Dependency}

Set the first row (i.e., wind speed equals to 0.5) as index $100 \%$ in Table III, we can obtain Table IV. The purpose is to show how much $\eta$ changes due to only wind speed in percentage at different temperatures. It is shown that the ratio varies little at the same wind speed while temperature varies.

\section{B. Temperature Dependency}

The results illustrated in Table $\mathrm{V}$ has a similar approach as in Table IV, but with focus on validate the temperature contribution. The first result column in Table III (i.e., $35^{\circ} \mathrm{C}$ ) is set to index $100 \%$, even if the underlying value is different in each row. It is shown that the ratio varies little or is the same at the same temperature while the wind speed varies.
TABLE VI

ACCURACY OF $\eta(\%)$ At DifFERENT Wind SPEed AND TEMPERATURE APPLYING MODEL DLR 3

\begin{tabular}{l|l|r|r|r|r|r|r}
\hline$\eta(\%)$ & \multicolumn{7}{|c}{$T_{a}\left({ }^{\circ} \mathrm{C}\right)$} \\
\hline$v(m / s)$ & 35 & \multicolumn{1}{|c|}{5} & \multicolumn{1}{|c}{5} & \multicolumn{1}{c}{-5} & \multicolumn{1}{c}{-5} & \multicolumn{1}{c}{-15} & -25 \\
\hline 0.5 & 0.00 & 0.25 & 0.43 & 0.56 & 0.65 & 0.70 & 0.73 \\
1.0 & 0.43 & 0.67 & 0.85 & 0.98 & 1.06 & 1.11 & 1.13 \\
2.0 & 0.01 & -0.23 & -0.54 & -0.91 & -1.34 & -1.82 & -2.35 \\
3.0 & 0.00 & -0.26 & -0.61 & -1.03 & -1.51 & -2.05 & -2.66 \\
4.0 & 0.02 & -0.29 & -0.67 & -1.12 & -1.65 & -2.23 & -2.90 \\
5.0 & 0.02 & -0.31 & -0.72 & -1.20 & -1.76 & -2.39 & -3.10 \\
6.0 & 0.02 & -0.31 & -0.76 & -1.27 & -1.86 & -2.52 & -3.27 \\
\hline
\end{tabular}

TABLE VII

Comparison Between DLR 3 AND THe Comprehensive DLR Model

\begin{tabular}{ccccc}
\hline \hline Option & Optimal nr. & \multicolumn{3}{c}{ Profit(SEK) } \\
& & Aver. & Diff. & Diff.(\%) \\
\hline DLR 1 & 4 & $1.44 * 10^{7}$ & $-8.35 * 10^{4}$ & -0.57 \\
DLR 2 & 4 & $1.61 * 10^{7}$ & $7.29 * 10^{3}$ & +0.05 \\
DLR 3 & 5 & $2.12 * 10^{7}$ & $6.31 * 10^{3}$ & +0.03 \\
\hline
\end{tabular}

\section{Accuracy of the Proposed Model (DLR 3)}

Table VI shows the difference of the value of $\eta$ by applying DLR 3 model and the comprehensive model. The different between this model and the comprehensive model is increasing with the wind speed and the temperature. The model never overestimates more than $1.13 \%$ nor underestimates more than $3.27 \%$. And DLR 3 model is always conservative for the cases that wind speed (the speed hits the conductor hot spot) is higher than $1 \mathrm{~m} / \mathrm{s}$ and ambient temperature is lower than $35^{\circ} \mathrm{C}$. However, the differences are very small. It is also possible to be more conservative by scaling the perpendicular wind speed down. The results using the proposed DLR model (6) are compared with the comprehensive DLR model (2) and (3) by implementing in the same case study. It is shown in Table VII that the difference is small.

\section{CONCLUSION}

This paper provides two main contributions: 1) a general DLR calculation model on overhead lines; and 2) an economic optimization simulation model regarding wind power integration comparing DLR with more traditional approaches. The DLR calculation model is easy to apply by companies in daily operation where the dynamic line capacity is calculated as a function of static line capacity, wind speed and ambient temperature. The DLR calculation model is furthermore compared with comprehensive calculations that validate that the model is accurate enough. Furthermore it is concluded that the wind speed and temperature contributions can be modeled 
separately and then simply multiplied together to receive the total "DLR bonus."

The simulation model aims to calculate the value of applying DLR. The DLR concept implies that the capacity of a component varies dynamically as a function of external parameters, e.g., weather conditions and loading history. The output of this model facilitates connection of renewable electricity production (in this paper exemplified by wind power integration).

This paper also provides an application study where both the DLR calculation model and the simulation model are exemplified together and evaluated. Results from a large number of simulations are presented and analyzed. The results conclude that it is a significant economic potential of implementing DLR within WP integration. The two models proposed are simple to apply and can easily be developed by adding more complexity, for example by considering weather correlations. The simulation model is not limited to power lines, but can be expanded to include all kinds of system parts that act as bottlenecks in the transfer capacity. The DLR calculation model is, however, limited to overhead lines, but can in contrast be valuable to use within all kinds of DLR usage, not only wind power integration.

\section{REFERENCES}

[1] M. Nehrir, C. Wang, and V. Gerez, "Impact of wind power distributed generation on distribution systems," in Proc. 17th Int. Conf. Elect. Distrib. (CIRED), Barcelona, Spain, May 2003, pp. 1-5.

[2] (2009). "Smart grid system report," U.S. Department of Energy [Online]. Available: http://energy.gov/oe/downloads/2009-smart-gridsystem-report-july-2009

[3] D. R. Swatek, "An expected per-unit rating for overhead transmission lines," Int. J. Elect. Power Energy Syst., vol. 26, no. 4, pp. 241-247, May 2004.

[4] S. Abdelkader et al., "Dynamic monitoring of overhead line ratings in wind intensive areas," in Proc. Eur. Wind Energy Conf. (EWEC), 2009, pp. $1-10$.

[5] K. E. Holbert and G. T. Heydt, "Prospects for dynamic transmission circuit ratings," in Proc. IEEE Int. Symp. Circuits Syst. (ISCAS), vol. 3. Sydney, NSW, Australia, May 2001, pp. 205-208.

[6] A. Leite, C. Borges, and D. Falcao, "Probabilistic wind farms generation model for reliability studies applied to Brazilian sites," IEEE Trans. Power Syst., vol. 21, no. 4, pp. 1493-1501, Nov. 2006.

[7] M. Khaki, P. Musilek, J. Heckenbergerova, and D. Koval, "Electric power system cost/loss optimization using Dynamic Thermal Rating and linear programming," in Proc. IEEE Elect. Power Energy Conf., Halifax, NS, Canada, Aug. 2010, pp. 1-6.

[8] P. Schell, J.-J. Lambin, B. Godard, H.-M. Nguyen, and J.-L. Lilien, "Using dynamic line rating to minimize curtailment of wind power connected to rural power networks," in Proc. 10th Int. Workshop Large-Scale Integr. Wind Power Syst., Aarhus, Denmark, Oct. 2011, pp. 1-5.

[9] T. Ringelband, M. Lange, M. Dietrich, and H.-J. Haubrich, "Potential of improved wind integration by dynamic thermal rating of overhead lines," in Proc. IEEE Bucharest PowerTech, Bucharest, Romania, Jun./Jul. 2009, pp. 1-5.

[10] L. Ochoa, L. Cradden, and G. Harrison, "Demonstrating the capacity benefits of dynamic ratings in smarter distribution networks," in Proc. Innov. Smart Grid Technol. (ISGT), Gaithersburg, MD, USA, 2010, pp. 1-6.

[11] F. Josue et al., "Transformer hot-spot temperature estimation for shorttime dynamic loading," in Proc. Int. Conf. Cond. Monit. Diagn. (CMD), Bali, Indonesia, Sep. 2012, pp. 217-220.

[12] D. Douglass. (2011). Dynamic Rating of Overhead Lines [Online]. Available: http://ieee-tpc.org/ieee_tutorials/ IEEE-Tutorial_DynamicthermalLineRatings_26July2011.pdf

[13] IEEE Standard for Calculating the Current-Temperature of Bare Overhead Conductors, IEEE Std 738-2006 (Revision of IEEE Std 738-1993), 2007, pp. c1-c59.
[14] "Engineering recommendation P27: Current rating guide for high voltage overhead lines operating in the UK distribution system," Energy Networks Association, London, U.K., 1986.

[15] E. Fernandez, I. Albizu, M. Bedialauneta, A. Mazon, and P. Leite, "Dynamic line rating systems for wind power integration," in Proc. IEEE Power Eng. Soc. Conf. Expo. Afr. (PowerAfrica), Johannesburg, South Africa, Jul. 2012, pp. 1-7.

[16] T. Goodwin and C. Smith, "Smart grid demonstration project-Dynamic line rating (DLR) - Oncor electric delivery," ERCOT Region Operations Training Seminar, TX, USA, 2011.

[17] A. Michiorri, R. Currie, and P. Taylor, "Dynamic line ratings deployment on the Orkney smart grid," in Proc. 21th Int. Conf. Elect. Distrib. (CIRED), Frankfurt, Germany, Jun. 2011.

[18] T. Yip, C. An, G. Lloyd, M. Aten, and B. Ferri, "Dynamic line rating protection for wind farm connections," in Proc. CIGRE/IEEE PES Joint Symp. Integr. Wide-Scale Renew. Resour. Power Del. Syst., Jul. 2009, pp. $1-5$.

[19] M. Schmale, R. Puffer, and M. Heidemann, "Dynamic ampacity rating of conductor bars in highly loaded substations," in Proc. 22nd Int. Conf. Exhibit. Elect. Distrib. (CIRED), Stockholm, Sweden, Jun. 2013, pp. $1-4$.

[20] C. J. Wallnerström, "On incentives affecting risk and asset management of power distribution," Ph.D. dissertation, Dept. Electromagn. Eng., KTH Royal Institute of Technology, Stockholm, Sweden, 2011.

[21] Y. Huang. (2014). Hourly Average Temperature in Mid-West Sweden [Online]. Available: https://kth.box.com/s/o9265aol7qetjhod5k31

[22] S. Talpur, "Dynamic line rating implementation as an approach to handle wind power integration," Master thesis, KTH Royal Inst. Technol., Stockholm, Sweden, Jun. 2013.

[23] (2014, Feb. 24). Nord Pool Spot [Online]. Available: http://www.nordpoolspot.com/

[24] (2014, May 10). Swedish National Grid [Online]. Available: http://www.svk.se/Start/English/

Carl Johan Wallnerström (GSM'06-M'11) was born in Stockholm, Sweden, in 1980. He received the M.Sc. and Ph.D. degrees in electrical engineering from the KTH Royal Institute of Technology, Stockholm, Sweden, in 2005 and 2011, respectively.

He completed post-doctoral projects in risk management applied to power distribution and dynamic rating, in 2012 and 2013, respectively. He is currently involved in a smart grid analysis project commissioned by the Swedish Coordination Council for Smart Grid.

Yalin Huang (GSM'12) was born in Hubei, China, in 1987. She received the B.Sc. degree in electrical engineering from the South China University of Technology, Guangzhou, China, in 2009, and the M.Sc. degree in electrical engineering from the KTH Royal Institute of Technology, Stockholm, Sweden, in 2011, where she is currently pursuing the Ph.D. degree.

Her current research interests include risk analysis methods and network regulation for electric power systems with integrated distributed generation.

Lennart Söder (M'91-SM'10) was born in Solna, Sweden, in 1956. He received the M.Sc. and Ph.D. degrees in electrical engineering from the KTH Royal Institute of Technology, Stockholm, Sweden, in 1982 and 1988, respectively.

He is currently a Professor of Electric Power Systems, KTH Royal Institute of Technology. He also works with projects concerning deregulated electricity markets and integration of wind power. 